\title{
Life as a physician in general internal medicine
}

\author{
Jacqui Wise
}

London, UK

General internal medicine is mainly a hospital inpatient specialty, with some outpatient work in rapid access clinics and for patient follow-up. Work is usually based in an acute medical unit, medical admissions unit, or on the wards.

Doctors specialising in general internal medicine are part of the acute medical care workforce which includes those who practise acute medicine, cardiology, diabetology, gastroenterology, geriatrics, renal medicine, respiratory medicine, and rheumatology.

Most doctors who practise general internal medicine are also trained in another specialty or subspecialty. Some may also combine general internal medicine with academic research or a non-clinical role.

\section{Nature of the work}

General internal medicine physicians staff the acute medical on-call rota and provide early consultant review duties on the acute medical unit with acute physician colleagues. The work involves diagnosing and treating a wide spectrum of diseases and conditions and referring for specialist opinion and care as needed.

Patients admitted to the acute medical unit who are not triaged to early specialist care but need hospitalisation are usually transferred to another ward within 24 hours of admission. The aim is to transfer the patient to a specialist ward best suited to their needs, but they may be transferred to the first available hospital bed if there is diagnostic uncertainty or if a specialty bed is not available. General internal medicine physicians manage these patients alongside specialty patients or on general medical wards. They often manage patients who have multiple comorbidities, including elderly patients with frailty and dementia.

Some out of hours work is required, and over $70 \%$ of consultants say they are routinely on-call at weekends.

\section{A typical day}

Much of the time is spent reviewing newly admitted patients, continuing treatment of the most unwell, or providing advice and care for those who have developed an acute illness while admitted for another reason, such as surgery or childbirth. Some time may also be taken up with outpatients who may be referred with chest pain or other urgent symptoms.

Most medical inpatients needing a stay of more than 48 hours are older patients with complex acute illness on top of at least one chronic disease. These patients often come under the care of physicians in general internal medicine who can provide them with continuing holistic care while ensuring that specialist input is requested when necessary. A broad range of patients are cared for under the umbrella of general internal medicine ranging from the young fit person with a severe acute illness to the frail elderly person with multiple comorbidities.

Physicians in general internal medicine need to be skilled in diagnostic reasoning as they will encounter patients with a wide spectrum of medical conditions that present acutely to hospital emergency departments and acute medical units. Part of the skillset is knowing when it is appropriate to refer for specialist opinion and care. Some of the day may be taken up with ordering chest $\mathrm{x}$ rays or blood tests and chasing up results.

Doctors in general internal medicine have expertise in managing patients with complex medical problems involving multiple symptoms and conditions, including patients with frailty and dementia. As well as prescribing drugs for acute and long term conditions, they also carry out interventions and procedures including advanced cardiopulmonary resuscitation, direct current cardioversion, temporary cardiac pacing, insertion of venous lines, aspiration of fluid from the chest and abdomen, insertion of drainage catheters into the chest and abdomen, and lumbar puncture.

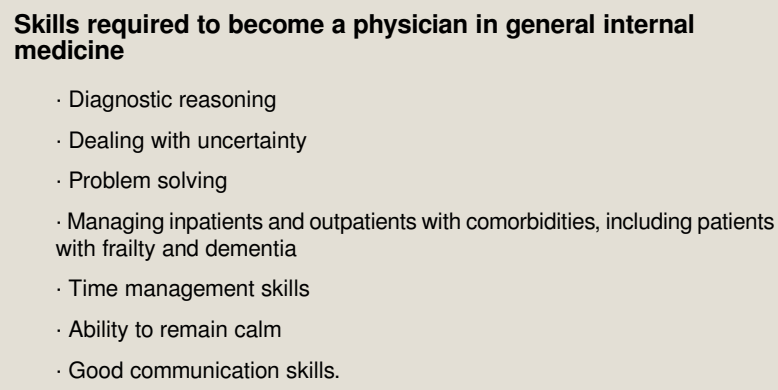

\section{Attractions of being a physician in general internal medicine}

The opportunity to see a wide range of illness and manage complex cases

Treating acutely ill people and saving lives can be rewarding There are opportunities to get involved in teaching, training, or research Can be combined with another specialty or subspecialty

The specialty is well suited to flexible training and working patterns. According to Health Education England a significant proportion of trainees are training less than full time. 
Challenges of being a physician in general internal medicine

Busy shifts can be tiring

Managing acutely ill people under pressure can be stressful

Increasing volume of work particularly with care for the ageing population

Generalists sometimes complain that other doctors perceive them to

have a lower status than specialists

The breadth of medical knowledge required can be challenging. 